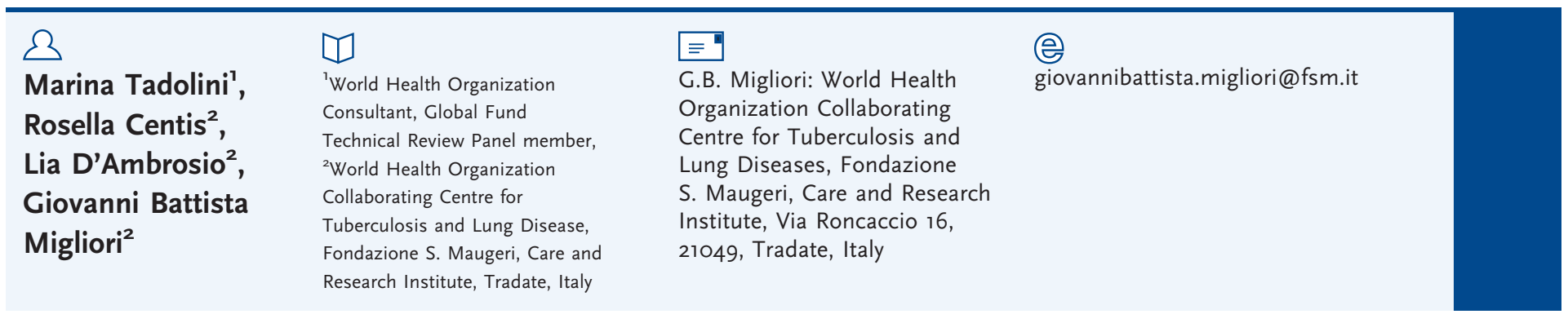

\title{
TB and MDR-TB: what is new in 20I2?
}

\section{Educational aims}

$\odot$ To understand the need for new diagnostics for tuberculosis (TB) and describe the role of GeneXpert

- To describe to what extent drug resistance affects clinical management of multi/ extensively drug resistant-TB, and the importance of having new drugs to manage these difficult-to-treat cases

๑ To describe the current evidence on TB/HIV collaborative activities

- To discuss the importance of providing a public-health response to reach TB elimination through a new post-2015 strategy and to protect new drugs through rational use of treatment regimens

\section{Summary}

In 2007, the 125th anniversary of the discovery of Mycobacterium tuberculosis by Robert Koch followed a particularly significant year in the history of tuberculosis (TB) as, in the same year, the World Health Organization and other international organisations published a series of pillar documents and guidelines that set the pathway to improve the control of TB at the global level. Among these, we can mention the Stop TB Strategy, the guidelines on drug-resistant TB management, the Global Plan to Stop TB 2006-2015 and the International Standard of TB Care. Despite the positive results obtained with the implementation of the new recommendations, some crucial gaps remained urgent, such as the need for innovative diagnostic tools for the prompt diagnosis of TB, the rapid identification of drug-resistant strains and the development of new drugs to combat the increasing number of drug-resistant forms of TB.

This year, 130 years after Robert Koch's discovery, we can recognise that some of these requests have been met. This article will describe the progresses made in different fields including: laboratory diagnosis and the experience with Xpert ${ }$ MTB/RIF assay; multi/extensively drug resistant-TB management and development of new promising drugs; implementation of TB/HIV collaborative activities.
Statement of Interest None declared.

HERMES syllabus link: Module A.1.5, A.1.7,

A.1.8, A.1.9;

Module B.4;

Module $17,12,13,17,18,19$ 
In addition, it will briefly describe the message of the European Union Standards for TB Care, recently developed by the European Respiratory Society and the European Centre for Disease Prevention and Control.

A strong public-health response is now needed to translate the latest recommendations into practice, make good use of the new diagnostic tools and drugs, improve the quality of TB and TB/HIV service worldwide and minimize the development of new cases of MDR-TB, through the correct clinical management of newly diagnosed, drug susceptible TB cases.

\section{Introduction}

On March 24th, 1882, Robert Koch discovered the tuberculosis (TB) bacillus (figs 1 and 2). In 2007, the 125th anniversary of the discovery of Mycobacterium tuberculosis was celebrated in different ways in different countries as an event that was perceived as one of the major achievements for the international medical community. The European Respiratory Society (ERS) celebrated the anniversary with an editorial, which is still the most cited in the European Respiratory Journal [1].

The reasons why this editorial has been so widely read is that, on top of the historical celebration, it highlighted several core messages, which are still of relevance.

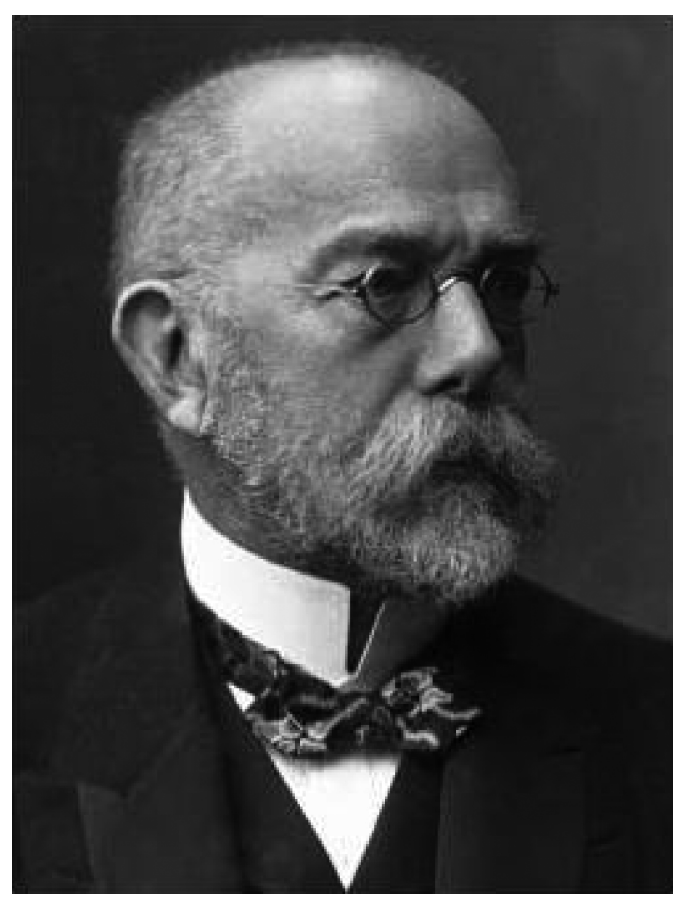

Figure 1

Robert Koch.

\section{The first message is clinical}

By emphasising the continuing importance of Koch's direct smear microscopy (a technique which is over 130 years old), the critical need for new diagnostics becomes more obvious. Of course, we do not have direct smear only, as culture (on solid and liquid media) and drug susceptibility testing have also been developed in the meantime [2]. But, unfortunately, in 2007, TB diagnosis was based only on dated techniques.

The recent availability of a new diagnostic tool, able to allow diagnosis of TB and resistance to rifampicin in one hour and 45 minutes, the Xpert ${ }^{\circledR}$ MTB/RIF assay (GeneXpert; Cepheid, Sunnyvale, CA, USA), is opening a new era in TB diagnostics [2-6]. We will describe the Xpert $_{\mathbb{R}}$ MTB/RIF assay later.

Furthermore, the editorial presented the recently described extensively drug-resistant (XDR) TB to the general public [1], underlining the limited evidence available and the urgent need for further surveillance, research and control [7]. We will describe below the core information on this severe form of disease, as well as the key publications which informed the recently published guidelines on multidrug resistant (MDR)-TB management [8].

The second message is one of public health

In 2006, almost 125 years after the Robert Koch's discovery, the new Stop TB Strategy was launched by the World Health Organization (WHO). This followed intensive exploration and discussion with TB control programme managers in high-burden countries, and partner organizations, including technical agencies and donors [9, 10]. The Stop TB Strategy built on the directly observed treatment, short-course (DOTS) strategy, launched by WHO in 1995. The DOTS strategy (consisting of five core 
elements: government commitment; diagnosis through microscopy; standardised and supervised treatment; uninterrupted drug supply; and regular programme monitoring) made a major contribution to improving global TB control [11], with more than 22 million patients treated under DOTS-based services [12, 13]. 11 years after its launch, the DOTS strategy was revisited to tackle the new challenges and make explicit five additional components to be implemented in order to reach the 2015 Millennium Development Goals (MDGs) relevant to TB control. Therefore, the Stop TB Strategy consisted of the following elements: 1) pursuing high-quality DOTS expansion and enhancement; 2) addressing TB/HIV, MDR-TB and other challenges; 3) contributing to health system strengthening; 4) engaging all care providers; 5) empowering people with TB and communities; and 6) enabling and promoting research [13].

It is useful, for educational purposes, to describe briefly the global targets for TB control. (fig. 3).

The MDG relevant to TB is MDG 6: it is aimed at combating HIV, malaria and other diseases. Goal 6 , Target 8 is to have halted and begun to reverse incidence of the disease by 2015 . The interpretation of Target 8 is that the incidence of all forms of TB should be falling by 2015 , and two indicators have been defined for this target: TB prevalence and deaths (Indicator 23), and the proportion of cases detected and successfully treated under the DOTS strategy (Indicator 24) [13].

The Stop TB Partnership targeted: a) reducing the prevalence and death rate by $50 \%$ compared with their level in 1990 by 2015 ; and b) eliminating TB by 2050 (fig. 3) [11].

In 2006, the new Global Plan to Stop TB, 2006-2015 was launched, describing strategies, financial requirements and existing gaps to reach the MDGs in all regions of the world.

Those who are not TB experts will ask whether these targets and indicators have been reached already or whether they will be reached by 2015 .

According to the latest Global TB Report (2011) published by WHO, the world and most regions, except Africa, are on track to achieve the MDG target of decreasing tuberculosis incidence by $2015[9,10]$. As far as the TB mortality is concerned, deaths from tuberculosis have fallen by $40 \%$ globally since 1990 , and achievement of the $50 \%$ reduction target by 2015 is likely. Despite the fact that TB

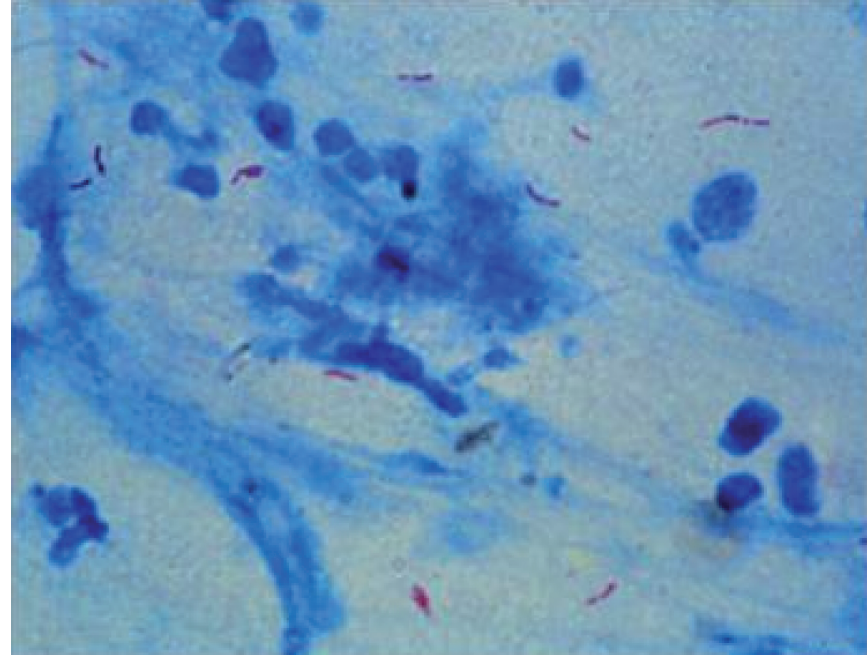

Figure 2

Direct sputum smear examination.

prevalence rates are also falling, the target of halving 1990 rates by 2015 is unlikely to be achieved, except in the Americas and the Western Pacific region [9]. In contrast, elimination of TB by 2050 is not achievable with the current trend, although substantial progresses might be made in the next few decades.

Strategy, objectives, targets and indicators had all been targeted toward 2015. That is why WHO and partners have already started discussing the steps to be undertaken after 2015. This article will go on to describe the lines of the ongoing discussion, as developed by Strategic and Technical Advisory Group for Tuberculosis (STAG-TB), the core advisory body convened once a year by WHO.

Global TB Control Targets (1) THO THE TSOP TB

\begin{tabular}{|c|c|}
\hline \multicolumn{2}{|c|}{$\begin{array}{l}\text { UN Millennium } \\
\text { Development Goals }\end{array}$} \\
\hline \multicolumn{2}{|c|}{ 2015: Goal 6: Combat HIV/AIDS, malaria and other diseases } \\
\hline Target 6c: & $\begin{array}{l}\text { Halt and begin to reverse the incidence of malaria and } \\
\text { other major diseases }\end{array}$ \\
\hline Indicator 6.9: & $\begin{array}{l}\text { Incidence, prevalence and deaths rates associated with } \\
\text { TB }\end{array}$ \\
\hline
\end{tabular}

Indicator 6.10: Proportion of TB cases detected and cured under DOTS

\section{Stop TB Partnership}

2015: Reduce prevalence and death rates by $50 \%$, compared with their levels in 1990

2050: Reduce the global incidence of active TB cases to $<1$ case per million population per year

Figure 3

Global TB control targets. 


\section{The third message is educational}

In 2006, the International Standards of TB Care (ISTC) were published and widely distributed, to guide both public and private healthcare providers to offer quality DOTS services [14]. In the same year, the WHO guidelines on drug-resistant TB management were also published, stressing the importance of proper use of second-line drugs required to treat drug-resistant and MDR-TB cases [15].

Although the year 2006 witnessed so many important TB events, several TB studies, documents and guidelines were published in the last two years. In particular, this article will summarise the core content of three documents, the ERS-European Centre for Disease Prevention and Control (ECDC) jointly developed European Standards for TB Care (representing the European Union adaptation of the ISTC) $[16,17]$, the 2011 WHO MDR-TB guidelines (representing an update of previous edition of WHO guidelines) [18] and the updated policy on TB/HIV collaborative activities, published by WHO in $2012[14,19,20]$.

\section{Clinical diagnosis of TB and the role of GeneXpert}

The diagnosis of TB is based on microbiological confirmation of the clinical picture. In other words, all TB suspects should be tested for TB by quality-assured bacteriology, as stated by the Stop TB strategy. Worldwide, the most accessible test for pulmonary TB diagnosis is sputum smear microscopy, followed by culture, now available both on solid and in liquid media. This constitutes the gold standard for TB diagnosis. Beside smear microscopy and culture, drug susceptibility testing (DST) has become more and more important to detect the bacilli resistance pattern to first- and second-line drugs [10].

Conventional methods for $M$. tuberculosis culture and DST are slow and cumbersome, requiring sequential procedures for the diagnosis. During this time, patients may be inappropriately treated, drug resistant strains may continue to spread, and amplification of resistance may occur. Therefore, the development, piloting and rolling-out of novel technologies providing rapid TB identification and detection of drug resistant strains are the current focus of TB research [6]. In particular, research on the Xpert巴 MTB/RIF assay has proliferated subsequent to $\mathrm{WHO}$ endorsement at the end of December 2010 [2-6]. By July 2012, >65 peer-reviewed publications had come out, testifying the global interest for the new test.

The Xpert system looks like a coffee machine, but it is able to tell us in 1 hour and 45 minutes whether M. tuberculosis is present, as well as whether resistance to rifampicin can be detected (fig. 4). The self-contained, cartridge-based test does not require much training, or advanced laboratory facilities or biosafety measures. The GeneXpert platform, launched in 2004, was designed to simplify molecular testing by integrating and automating the three key processes (sample preparation, amplification and detection) required for real-time PCR-based molecular testing [2-6]. Core components of the system include the instrument, a personal computer, a barcode scanner and the software (fig. 4). Single-use disposable cartridges containing lyophilised reagents, buffers and washes are used for specific diseases. Target detection and characterisation is performed in real time using a six-colour laser detection device.

The Xpertß MTB/RIF assay employs five unique nucleic acid hybridisation probes (molecular beacon), each labelled with a differently coloured fluorophore responding to a specific target sequence within the rpoB gene of Mycobacterium tuberculosis. 95\% of mutations associated with rifampicin resistance occur in an 81-base pair core region of the rpoB (a bacterial RNA polymerase) gene and, together, these five molecular beacons encompass the

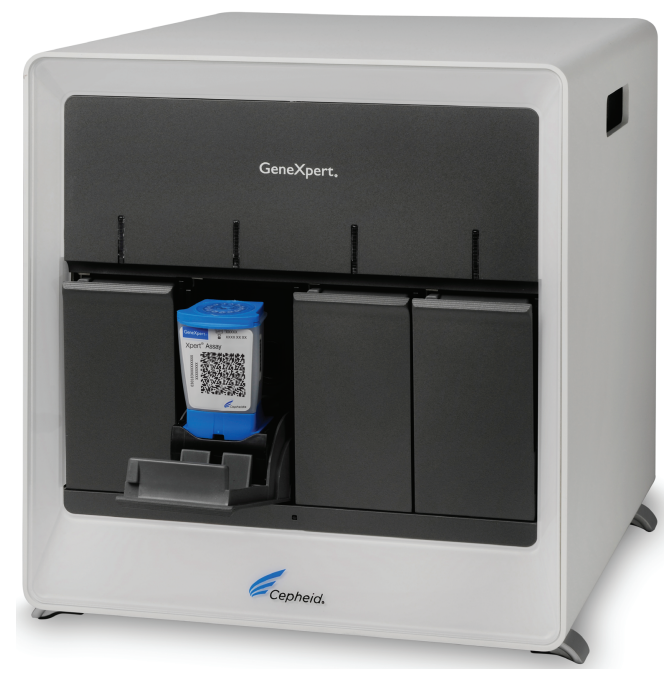

Figure 4

GeneXpert. (c) Cepheid 
entire core region. The generation of all five fluorescent colours during PCR amplification indicates the presence of rifampicin-susceptible M. tuberculosis, while any mutation in the core region prevents the binding of the respective molecular beacon, resulting in the absence of colour and indicating rifampicin resistance.

The main advantage of the GeneXpert system is to provide a rapid, simultaneous diagnosis of both TB and rifampicin resistance. BOHEME and colleagues [3, 4] and others $[2,5,6]$ have shown that GeneXpert is highly specific, diagnosing $98.2 \%$ of sputum smear positive patients and $72.5 \%$ of smear negative, culturepositive patients (which increased to $90.2 \%$ if three tests were used), and correctly identifying $97.6 \%$ of the rifampicin-resistant bacilli.

GeneXpert has the potential to become "the" diagnostic test for TB, it is used at present for pulmonary (sputum smear positive and negative) TB disease. Initial results indicate that the main problem to clarify is how to interpret positive results in different epidemiological settings, as the positive predictive value (PPV) of the assay in low-TB incidence settings is relatively low in detecting rifampicin resistance. In addition, studies are accumulating to evaluate its performance in extrapulmonary and paediatric TB.

One of the distinct advantages of Xpert $\mathrm{R}_{\mathbb{R}}$ MTB/RIF is its suitability for use at district and sub-district level and the technology should therefore not be restricted to central/reference laboratory level only.

On top of further studies providing evidence of the feasibility, efficacy and costeffectiveness, the new priority is to determine how to locate GeneXpert within existing national diagnostic algorithm.

The European Standards of TB Care (ESTC) [16] clearly considers the use of rapid diagnostic methods and GeneXpert as a must in EU countries, as this significantly reduces the time to diagnosis and the time to treatment. In other words, it contributes to reduce significantly the infectiousness period of the cases and, therefore, the potentiality for transmission of TB bacilli within the community.

In addition, the early detection of rifampicin resistance, which is a reliable proxy of multidrug resistance (pending the limitations described above), allows the referral of the "potential" MDR-TB patient to a specialised centre, so that proper management can be rapidly implemented [22-24].

\section{Clinical management of M/XDR-TB, with the availability of new drugs}

The prevalence of drug-resistant TB is alarmingly high, particularly in the countries belonging to the former Soviet Union, as summarised in figures 5 and 6 . The new "global record" of MDR-TB prevalence was recently notified in Belarus [25] with 35.3\% MDR-TB among new cases and $76.5 \%$ MDR-TB among previously treated cases. It clearly demonstrated how far case mismanagement can affect the chances to control (and eventually eliminate) the disease [23].

As a consequence, additional levels of drug resistance beyond XDR-TB (XDR-TB is defined as resistance to at least rifampicin and isoniazid (the definition of MDR-TB), in addition to resistance to any fluoroquinolone, and to at least one of the three following injectable drugs used in anti-TB treatment: capreomycin, kanamycin and amikacin) have been described in Italy, Iran and India [26-28] and, provisionally, named "total drug resistance" (e.g. cases harbouring Mycobacterium tuberculosis strains with resistance to all known drugs).

Although WHO has not endorsed this definition, due to the difficulties to agreeing on the panel of drugs which should define it [29], these super-resistant strains are circulating, projecting affected patients back to the pre-antibiotic era when effective drug against the bacilli were not available.

In order to effectively tackle the MDR-TB challenge, ESTC [16] still emphasises the importance of ensuring rapid diagnosis and appropriate treatment of MDR-TB cases.

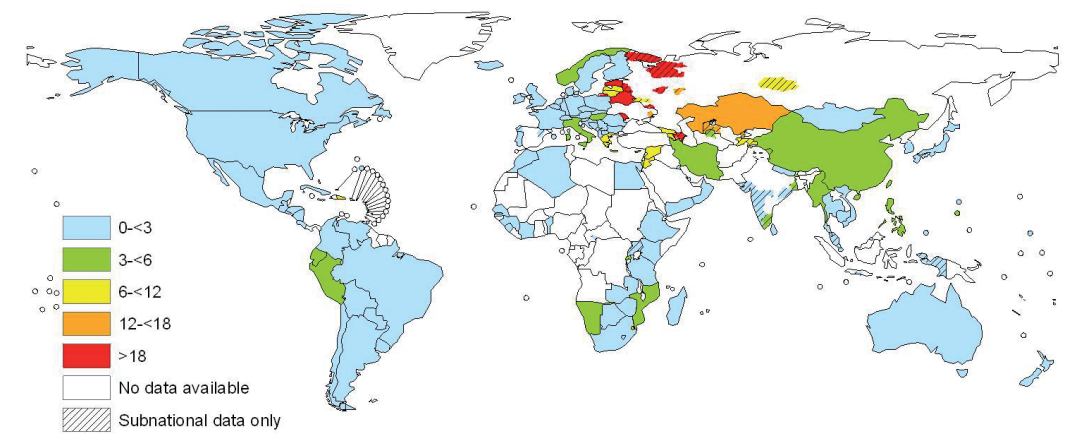

Figure 5

Proportion of MDR among new TB cases. Latest available data, 1994-2010. Reproduced from [21] with permission from the publisher. 


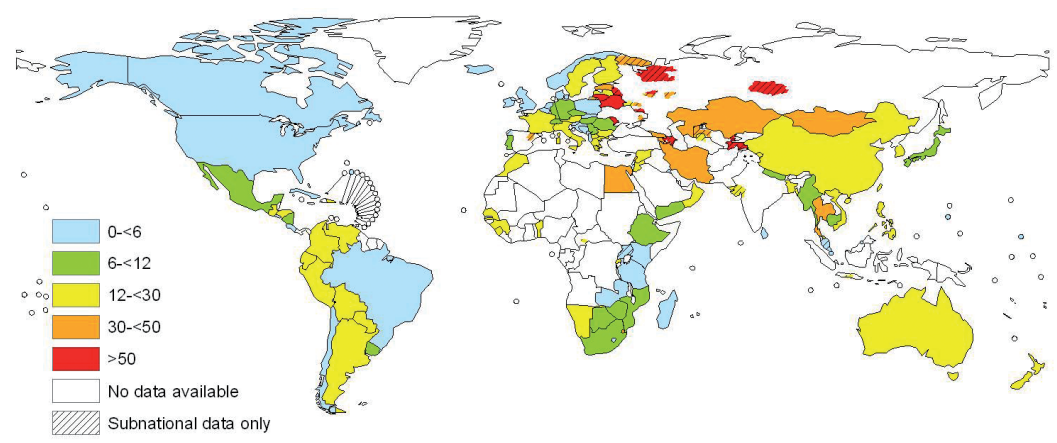

Figure 6

Proportion of MDR among previously treated TB cases. Latest available data, 1994-2010. Reproduced from [21] with permission from the publisher.

\section{What are the standards?}

They have been defined as "a living document that will be revised as technology, resources and circumstances change", being designed to complement existing national or international guidelines.

There are specific characteristics of the countries of the EU justifying the need for a European-specific adaptation of the ISTC described above into ESTC.

Europe is a heterogeneous setting, with low-, intermediate- and high-level TB countries represented, and varying levels of MDR-TB and TB/HIV co-infection. TB services are usually integrated and merged within the health system, although vertical programmes are still in place in some countries. European countries are committed to pursuing elimination, sharing a common platform (based on the Wolfheze documents and the EU Framework action plan) and the ECDC-driven surveillance system.
The core messages included in the ESTC are summarized in table 1.

In 2011, WHO published a revision of the previous guidelines on MDR-TB management, taking advantage of a recent study on a large MDR-TB cohort of about 10,000 cases $[18,30]$, summarised in table 2.

At the same time, the search for new drugs has become more and more urgent.

One direction followed by researchers was to evaluate the possible role of drugs in TB treatment although little was known on their efficacy, safety and tolerability. Among these drugs, linezolid (effective but unfortunately toxic [31]) and meropenem's activity seems to be promising [32].

The main directions followed development of the three most promising compounds in the development pipeline (delamanid, bedaquiline and PA-824) [33-37].

Delamanid, in combination with a background regimen developed according to the WHO guidelines [33], is associated with an increase in sputum-culture conversion at 2 months in patients with MDR-TB. Furthermore, it is able to increase treatment success and dramatically reduce mortality in these difficultto-treat cases [34].

In a recent multiple-agent combination study published in the Lancet $[35,37]$ the 14-day early bactericidal activity of Pa-824+moxifloxacin+pyrazinamide was found to be significantly higher than that of bedaquiline, bedaquiline+pyrazinamide, $\mathrm{Pa}-824+$ pyrazinamide and bedaquiline+Pa- 824 and comparable to that of the standard treatment regimen of

Table 1. Rationale for the four key components of the ESTC; adapted from [17]

Diagnosis The ESTC are based on TB suspects identified not only through sputum smear microscopy, but adapted to the EU/EEA setting including culture, DST (drug susceptibility testing) and rapid testing including rapid identification for rifampicin resistance. This is consistent with the culture-based definition used in the EU/EEA.

Treatment Given the availability of rapid diagnostic for TB and rifampicin resistance, the need to start MDR treatment from the beginning, under high-quality management conditions.

To ensure quality treatment monitoring based on culture.

HIV/TB and co-morbidities

Public health and prevention
Universal access to anti-retroviral treatment is available to all HIV infected as well as the capacity to detect all cases. This impacts on the management of provision of isoniazid preventive therapy (IPT).

Not only HIV infected but also individuals with a range of immunosuppressive conditions (such as TNF- $\alpha$ antagonist, corticosteroid, cancer treatment, diabetes, intravenous drug use and alcohol abuse) should be evaluated for IPT and proper follow-up for TB.

In absence of vertical TB programmes and with the full integration of services, healthcare providers are assuming an important responsibility for ensuring key public health and infection control functions related to detection and prevention of TB. 
isoniazid, rifampicin and pyrazinamde with streptomycin or ethambutol.

This finding opens the way to a potential new vision of treatment, in which a single, universal regimen not built around rifampicin and isoniazid (e.g. the two drugs whose resistance defined MDR-TB) would (hopefully) be able in the near future to treat both drug susceptible and drug-resistant TB cases [37].

\section{TB/HIV}

In 2004, WHO published an interim policy on collaborative TB/HIV activities for immediate guidance to countries on actions to decrease the dual burden of TB and HIV [38]. Since then, additional evidence has been generated from randomised controlled trials, observational studies, operational research and best practices from programmatic implementation of the collaborative TB/HIV activities recommended by the policy. In 2012, WHO published the updated policy on collaborative TB/HIV activities, to summarise the current evidence and experience in implementing collaborative TB/ HIV activities [19] (table 3).

The new policy follows the same framework as the 2004 interim policy document, structuring the activities under three objectives: establishing and strengthening mechanisms for integrated delivery of TB and HIV services; reducing the burden of TB among people living with HIV and reducing the burden of HIV among people with presumptive TB (that is, people with signs and symptoms of TB or with suspected TB) and diagnosed TB.

Under the first objective, the updated policy emphasises the need to establish mechanisms for delivering integrated TB/ HIV services, preferably at the same time and location. Integrated TB/HIV services should be also offered as part of other health programmes, such as maternal and child health, harm-reduction services and prison health services. Monitoring and evaluation of collaborative TB/HIV activities should be done within one national system using standardised indicators and reporting and recording formats. TB prevalence surveys should include HIV testing, and HIV surveillance systems should incorporate TB screening as routine practice. National and local targets for collaborative TB/HIV activities should be jointly set, for example through the national TB/HIV coordinating body and national consultations. Long- and mediumterm national TB/HIV strategic plans should be developed to scale up activities nationwide. National HIV programmes and TBcontrol programmes should establish linkage and partnerships with a broad platform of governmental, nongovernmental and community organisations for programme development, implementation and monitoring of TB/HIV collaborative activities.

The second objective describes the interventions to reduce the burden of TB among people living with HIV, which include the early provision of antiretroviral therapy (ART) for people living with HIV and the "three Is for HIV/TB": intensified TB case finding followed by high-quality antituberculosis treatment; isoniazid preventive therapy (IPT); and infection control for TB. The policy recommends that all people living with HIV should be regularly screened for TB using a simple clinical symptom-based algorithm consisting of: a) current cough; b) fever; c) weight loss; or d) night sweats. TB symptom screening should be performed at the time of initial presentation for HIV care and at every visit to a health facility or contact with a healthcare worker [39]. Screening for TB is important regardless of whether they have received or are receiving IPT or ART. Adults and adolescents living with HIV who report any one of the mentioned symptoms may have active TB and should be evaluated for TB.

Smear-negative pulmonary and extrapulmonary TB is common among people living with HIV and associated with poor treatment outcomes and excessive early mortality. If smear-negative pulmonary TB or extrapulmonary $\mathrm{TB}$ is suspected, diagnostic processes should be accelerated using all available and appropriate investigations, including mycobacterial culture [4O]. If available, WHO approved molecular tests (e.g. Xpert MTB/RIF) should be the primary diagnostic test for TB in people living with HIV [41]. In case of negative investigations and no improvement to parenteral antibiotics among seriously ill patients in HIV-prevalent settings, empirical anti-TB treatment should be initiated [40]. New TB patients living with HIV should receive a standard TB regimen containing 6 months of rifampicin $(2 \mathrm{HRZE} / 4 \mathrm{RH})$ on a daily schedule [42] and should be started on ART regardless of CD4 count as soon as possible within the first 8 weeks of anti-TB treatment [43]. 
Table 2. Changes to the recommendations on regimen composition between the 2008 and 2011 updates of WHO MDR-TB guidelines

2008 emergency update

Include at least four anti-TB drugs with either certain, or almost certain, effectiveness during the intensive phase of $T x$.

Consider adding more drugs in patients with extensive disease or uncertain effectiveness

\section{1 update}

Include at least four second-line anti-TB drugs likely to be effective as well as $Z$ during the intensive phase of Tx.

No evidence found to support the use of more than four second-line anti-TB drugs in patients with extensive disease. Increasing the number of second-line drugs in a regimen is permissible if the effectiveness of some of the drugs is uncertain.

The regimen should include $\mathrm{Z}$ and/or $\mathrm{E}$, one $\mathrm{FQ}$, one parenteral agent and second-line oral bacteriostatic anti-TB drugs (no preference of oral bacteriostatic second-line anti-TB drug was made).

E may be considered effective and included in the regimen if DST shows susceptibility

The regimen should include $Z$, a FQ, a parenteral agent, ethionamide (or prothionamide), and cycloserine, or else PAS if cycloserine cannot be used.

E may be used but is not included among the drugs making up the standard regimen.

Tx with group-5 drugs is recommended only if additional drugs are needed to bring the total to four

Group-5 drugs may be used but are not included among the drugs making up the standard regimen

Intensive phase minimum of 6 months (minimum 4 months after $\mathrm{C}$ conversion) for a total duration of minimum of 18 months after $\mathrm{C}$ conversion

Intensive phase minimum of 8 months for a total duration $\geqslant 20$ months

Z: pyrazinamide; E: ethambutol; FQ: fluoroquinolone; PAS: para-aminosalycilic acid ; C: capreomycin; Tx: treatment.

Administrative, environmental and personal protective measures against TB infection in healthcare facilities and congregate settings should be put in place. These measures include surveillance of HIV and TB among healthcare workers; relocation of health workers living with HIV from areas with high TB exposure; and provision of ART and IPT.

Under the third component, the updated policy recommends offering routine HIV testing not only to patients with presumptive or diagnosed TB but also to their partners and family members, as a means of reducing the burden of HIV in the general community. All TB patients who are found to be HIVpositive should be provided with co-trimoxazole preventive therapy (CPT), regardless of $\mathrm{CD}_{4}$ cell-count. Antiretroviral treatment should be given to all HIV-positive TB patients as soon as possible within the first 8 weeks of anti-TB treatment, regardless of their CD4 cell-count. HIV-positive TB patients with profound immunosuppression (e.g. CD4 count $<50$ cells $\cdot \mathrm{mm}^{-3}$ ) should receive ART immediately, within the first 2 weeks of initiating TB treatment. TB patients, their family and community members should be provided with comprehensive HIV prevention services.

Public health response to develop a post-2015 strategy

In June this year, WHO and partners began discussions to define the new post-2015 strategy of TB control and elimination. The perspective of TB elimination (the definitions of control and elimination are reported in table 4 ) has now become central in WHO policies, something several countries (including in the EU, as well as USA, Japan and Canada) had committed to as far back as 1991 [44-47].

Through TB control, we aim at reducing the incidence of TB infection and, consequently, of TB disease, based on early diagnosis and treatment of infectious cases of TB, so that we reduce as much as possible the transmission of TB bacilli within the community.

TB elimination is an additional strategy aimed at reducing the prevalence of TB infection, based on preventive treatment of latently TB infected individuals. By reducing the large pool of infected individuals, future cases of TB will be prevented as no TB disease will occur if we do not have infection first [5]. 
Table 3. WHO-recommended collaborative TB/HIV activities; reproduced from [19] with permission from the publisher

A. Establish and strengthen the mechanisms for delivering integrated TB and HIV services

A.r. Set up and strengthen a coordinating body for collaborative TB/HIV activities functional at all levels

A.2. Determine HIV prevalence among TB patients and TB prevalence among people living with HIV

A.3. Carry out joint TB/HIV planning to integrate the delivery of TB and HIV services

A.4. Monitor and evaluate collaborative TB/HIV activities

B. Reduce the burden of TB in people living with HIV and initiate early antiretroviral therapy (the Three I's for HIV/TB)

B.1. Intensify TB case-finding and ensure high quality antituberculosis treatment

B.2. Initiate TB prevention with isoniazid preventive therapy and early antiretroviral therapy

B.3. Ensure control of TB Infection in health-care facilities and congregate settings

C. Reduce the burden of HIV in patients with presumptive and diagnosed TB

C.1. Provide HIV testing and counselling to patients with presumptive and diagnosed TB

C.2. Provide HIV prevention interventions for patients with presumptive and diagnosed TB

C.3. Provide co-trimoxazole preventive therapy for TB patients living with HIV

C.4. Ensure HIV prevention interventions, treatment and care for TB patients living with HIV

C.5. Provide antiretroviral therapy for TB patients living with HIV

Initial agreement was built around three main pillars, including intensified and innovative TB care, development and enforcement of bold health-system and social development policies, and promotion and intensification of research and innovation [5].

While the first pillar will capture the core technical principles described in the DOTS and Stop TB Strategy (rapid diagnosis, screening of populations at risk, treatment and patient support including MDR-TB and $\mathrm{TB} / \mathrm{HIV}$, treatment of latently infected individuals), the second pillar will capture the necessary policies supporting these principles (integration of health services; universal access; improved vital statistics, infection control and rationale use of quality drugs; fight against social determinants). The third pillar finally will further stimulate research and rational use of new diagnostic, drugs and vaccines [5, 47].

A strong public health system is necessary to minimise development of new cases of MDR-TB, through the correct clinical management of newly diagnosed, drug susceptible TB cases. This comprehensive approach, ensuring rational use of drugs, will also protect the investment in developing the new drugs, and prevent the development of new drug resistances [48].

Table 4. TB control and elimination

Definitions

TB control

Strategy aimed at reducing the incidence of TB infection and, consequently, of TB disease, based on early diagnosis and treatment of infectious cases of TB. Fewer and fewer people in the community will be newly exposed to the contact with the bacilli and will develop the disease

Elimination

Point at which less than one infectious (sputum smear positive) case per 1,000,000 inhabitants emerges annually in the general population or when the prevalence of TB in general population is below $1 \%$ and continues to decrease. 
- Tuberculosis control is aimed at reducing the incidence of TB infection (and, consequently, of TB disease, based on early diagnosis and treatment of infectious cases of TB), while TB elimination, is an additional strategy aimed at reducing the prevalence of TB infection, based on preventive treatment of latent TB. TB elimination is challenging due to the high prevalence of drug resistant TB (particularly in the former Soviet Union Countries), largely due to case mismanagement and health systems drawbacks. As a result additional levels of drug resistance beyond XDR-TB are appearing.

- The new Xpertæ MTB/RIF assay (GeneXpert, Cepheid, Sunnyvale, CA, USA), is opening a new era in TB diagnostics allowing a rapid, simultaneous diagnosis of both TB and resistance to rifampicin in 1 hour and 45 minutes.

- New drugs will soon be available in TB treatment, including three promising compounds: delamanid, bedaquiline and PA-824. New strategies have been developed by WHO to respond to the core challenges (2012 updated Policy on collaborative TB/HIV activities, draft post-2015 Strategy towards elimination); the new EU Standards for TB Care have been developed jointly by ERS and ECDC.

\section{References}

1. Migliori GB, Loddenkemper R, Blasi F, et al. 125 years after Robert Koch's discovery of the tubercle bacillus: the new XDR-TB threat. Is "science" enough to tackle the epidemic? Eur Respir J 2007; 29: 423-427.

2. Centis R, D'Ambrosio L, Migliori GB. Control and elimination of tuberculosis: do we only need new diagnostics? ERS Buyers' Guide 2011; 104-111.

3. Boehme CC, Nabeta P, Hillemann D, et al. Rapid molecular detection of tuberculosis and rifampin resistance. N Engl J Med 2010; 363: 1005-1015.

4. Boehme CC, Nicol MP, Nabeta P, et al. Feasibility, diagnostic accuracy, and effectiveness of decentralised use of the Xpert MTB/RIF test for diagnosis of tuberculosis and multidrug resistance: a multicentre implementation study. Lancet 2011; 377: 1495-1505.

5. Strategic and Technical Advisory Group for Tuberculosis (STAG-TB). Report of the 12th meeting. WHO Headquarters, Geneva, Switzerland, June, 2012.

6. World Health Organization. Automated real-time nucleic acid amplification technology for rapid and simultaneous detection of tuberculosis and rifampicin resistance: Xpert Mtb/Rif System. Policy Statement. World Health Organization, Geneva, Switzerland, 2011. Document WHO/HTM/TB/2011.4

7. Migliori GB, Richardson MD, Lange C. Of blind men and elephants: making sense of extensively drugresistant tuberculosis. Am J Respir Crit Care Med 2008; 178: 1000-1001.

8. Falzon D, Jaramillo $E$, Schünemann HJ, et al. WHO guidelines for the programmatic management of drug-resistant tuberculosis: 2011 update. Eur Respir J 2011; 38: 516-528.

9. World Health Organization. Global tuberculosis control 2011. World Health Organization, Geneva, Switzerland, 2011. Document WHO/HTM/TB/2011.16.

10. Raviglione M, Marais B, Floyd $\mathrm{K}$, et al. Scaling up interventions to achieve global tuberculosis control: progress and new developments. Lancet 2012; 379: 1902-1913.
11. Stop TB Partnership and World Health Organization. The Global Plan to Stop TB 2006-2015. World Health Organization, Geneva, Switzerland, 2006.

12. World Health Organization. WHO Report 2006. Global tuberculosis control: surveillance, planning, financing. World Health Organization, Geneva, Switzerland, 2006. Document WHO/HTM/TB/ 2006.362

13. Raviglione MC, Uplekar MW. WHO's new Stop TB Strategy. Lancet 2006; 367: 952-955.

14. Tuberculosis Coalition for Technical Assistance. International Standards for Tuberculosis Care (ISTC) The Hague, Tuberculosis Coalition for Technical Assistance, 2006.

15. World Health Organization. Guidelines for the programmatic management of drug-resistant Tuberculosis. World Health Organization, Geneva, Switzerland, 2006. Document WHO/HTM/TB/ 2006.361

16. Migliori GB, Zellweger JP, Abubakar I, et al. European Union Standards for Tuberculosis Care. Eur Respir J 2012; 39: 807-819.

17. Migliori GB, Sotgiu G, Blasi F, et al. Towards the development of EU/EEA Standards for Tuberculosis Care (ESTC). Eur Respir J 2011; 38: 493-495.

18. World Health Organization. Guidelines for the programmatic management of drug-resistant tuberculosis - 2011 update. World Health Organization, Geneva, Switzerland, 2011. Document WHO/HTM/ $\mathrm{TB} / 2011.6$.

19. World Health Organization. WHO policy on collaborative TB/HIV activities: guidelines for national programmes and other stakeholders. World Health Organization, Geneva, Switzerland, 2012. Document WHO/HTM/TB/2012.1.

20. Tuberculosis Coalition for Technical Assistance. International Standards for Tuberculosis Care (ISTC), 2nd edn. The Hague, Tuberculosis Coalition for Technical Assistance, 2009. 
21. Zignol M, van Gemert W, Falzon D, et al. Surveillance of anti-tuberculosis drug resistance in the world: an updated analysis, 2007-2010. Bull World Health Org 2012; 90: 111-119.

22. Dheda K, Migliori GB. The global rise of extensively drug-resistant tuberculosis: is the time to bring back sanatoria now overdue? Lancet 2012; 379: 773-775.

23. Migliori GB, Sotgiu G, D'Ambrosio L, et al. TB and MDR/XDR-TB in European Union and European Economic Area countries: managed or mismanaged? Eur Respir J 2012; 39: 619-625.

24. Sotgiu G, D'Ambrosio L, Centis R, et al. TB and M/ XDR-TB infection control in European TB reference centres: the Achilles' heel? Eur Respir J 2011; 38: 1221-1223.

25. Skrahina A, Hurevich $H$, Zalutskaya A, et al. Alarming levels of drug-resistant tuberculosis in Belarus: results of a survey in Minsk. Eur Respir J 2012; 39: $1425-1431$.

26. Migliori GB, De laco G, Besozzi G, et al. First tuberculosis cases in Italy resistant to all tested drugs. Euro Surveill 2007; 12: E070517.1.

27. Velayati AA, Masjedi MR, Farnia P, et al. Emergence of new forms of totally drug-resistant tuberculosis bacilli: super extensively drug-resistant tuberculosis or totally drug-resistant strains in Iran. Chest 2009; 136: 420-425.

28. Udwadia ZF, Amale RA, Ajbani KK, et al. Totally drug resistant tuberculosis in India. Clin Infect Dis 2012; 54: 579-581.

29. Migliori GB, Centis R, D'Ambrosio L, et al. Totally drug-resistant and extremely drug-resistant tuberculosis: the same disease? Clin Infect Dis 2012; 54: 1379-1380.

30. Ahuja SD, Ashkin D, Avendano M, et al. Collaborative group for meta-analysis of individual patient data in MDR-TB. Multidrug resistant pulmonary tuberculosis treatment regimens and patient outcomes: an individual patient data meta-analysis of 9,153 patients. PLoS Med 2012; 9: e1001300.

31. Sotgiu G, Centis R, D'Ambrosio L, et al. Efficacy, safety and tolerability of linezolid containing regimens in treating MDR-TB and XDR-TB: systematic review and meta-analysis. Eur Respir J 2012 [Epub ahead of print DOI: 10.1183/o9031936.00022912].

32. De Lorenzo S, Alffenaar JW, Sotgiu G, et al. Efficacy and safety of meropenem/ clavulanate added to linezolid containing regimens in the treatment of $\mathrm{M} /$ XDR-TB. Eur Respir J 2012 [Epub ahead of print DOI: 10.1183/og031936.00124312].

33. Gler MT, Skripconoka V, Sanchez-Garavito E, et al. Delamanid for multidrug-resistant pulmonary tuberculosis. N Engl J Med 2012; 366: 2151-2160.

34. Skripconoka V, Danilovits M, Pehme L, et al. Delamanid improves outcomes and reduces mortal ity for multidrug-resistant tuberculosis. Eur Respir J 2012 [Epub ahead of print doi: 10.1183/ og031936.00125812].

35. Diacon $A H$, Dawson $R$, von Groote-Bidlingmaier $F$, et al. 14-day bactericidal activity of PA-824, bedaquiline, pyrazinamide, and moxifloxacin combinations: a randomised trial. Lancet 2012 [Epub ahead of print 10.1016/So140-6736(12)61080-O].

36. D'Ambrosio L, Centis R, Migliori GB. Rational use of TB drugs, present action to preserve future options. $N$ Engl J Med 2012; In press.

37. Migliori GB, Sotgiu G. Treatment of tuberculosis: had we turned the corner? Lancet 2012; 380: 955-957.

38. World Health Organization. Interim policy on collaborative TB/HIV activities. World Health Organization, Geneva, Switzerland, 2004. Document WHO/HTM/TB/2004.330.

39. Getahun $\mathrm{H}$, Kittikraisak W, Heilig CM, et al. Development of a standardized screening rule for tuberculosis in people living with HIV in resourceconstrained settings: individual participant data meta-analysis of observational studies. PLoS Medicine 2011; 8: 1, e1000391.

40. World Health Organization. Improving the diagnosis and treatment of smear-negative pulmonary and extrapulmonary tuberculosis among adults and adolescents: recommendations for HIV-prevalent and resource-constrained settings. World Health Organization, Geneva, Switzerland, 2007. Document WHO/HTM/TB/2007.379.

41. World Health Organization. Roadmap for rolling out Xpert MTB/RIF for rapid diagnosis of TB and MDRTB. Geneva, WHO, 2010.

42. World Health Organization. Treatment of tuberculosis: guidelines; 4th edn. Geneva, Switzerland: World Health Organization, Geneva, Switzerland, 2009. Document WHO/HTM/TB/2009.42O.

43. World Health Organization. Antiretroviral therapy for HIV infection in adults and adolescents: recommendations for a public health approach, 2010 revision. Geneva, World Health Organization, 2010.

44. Clancy L, Rieder HL, Enarson DA, et al. Tuberculosis elimination in the countries of Europe and other industrialized countries. Eur Respir J 1991; 4: 1288-1295.

45. Broekmans JF, Migliori GB, Rieder HL, et al. World Health Organization, International Union Against Tuberculosis and Lung Disease, and Royal Netherlands Tuberculosis Association Working Group. European framework for tuberculosis control and elimination in countries with a low incidence. Recommendations of the World Health Organization (WHO), International Union Against Tuberculosis and Lung Disease (IUATLD) and Royal Netherlands Tuberculosis Association (KNCV) Working Group. Eur Respir J 2002; 19: 765-775.

46. Veen J, Migliori GB, Raviglione M, et al. Harmonisation of TB control in the WHO European region: the history of the Wolfheze Workshops. Eur Respir J 2011; 37: 950-959.

47. Tadolini M, Migliori GB. Tuberculosis control and elimination. Eur Respir Mogr 2012; 58: 243-253.

48. Migliori GB, Langendam MW, D'Ambrosio L, et al. Protecting the TB drug pipeline. Stating the case for the rational use of Fluoroquinolones. Eur Respir J 2012 [Epub ahead of print DOI: 10.1183/ og031936.00036812]. 